\title{
A SOLUTION OF THE QUANTUM KNIZHNIK ZAMOLODCHIKOV EQUATION OF TYPE $C_{n}$
}

\author{
KATSUHISA MIMACHI
}

\begin{abstract}
We construct a solution of Cherednik's quantum Knizhnik Zamolodchikov equation associated with the root system of type $C_{n}$. This solution is given in terms of a restriction of a $q$-Jordan-Pochhammer integral. As its applicaton, we give an explicit expression of a special case of the Macdonald polynomial of the $C_{n}$ type. Finally we explain the connection with the representation of the Hecke algebra.
\end{abstract}

\section{INTRODUCTION}

We study the quantum Knizhnik Zamolodchikov (QKZ) equation ([2|) associated with the root system of type $C_{n}$. A solution to this equation is found by means of a restriction of the $q$-Jordan-Pochhammer integral.

A solution of the QKZ equation of type $A_{n-1}$ is given in [14]. Since the appearance of that work, however, there has been no progress in the study of the QKZ equation for other types of root systems with regard to the determination of solutions. This paper is devoted to such a task.

To construct our solution, we exploit a family of rational functions which would correspond to a basis of the $q$ de Rham cohomology attached to the integrand. This turns out to be a natural basis for the representation of the Hecke algebra $H(W)$ through the Lusztig operator $T_{i}$.

Next, as a byproduct of our investigation, we obtain an integral representaion of the special case of an eigenfunction associated with the Macdonald operator of the $C_{n}$ type. In particular, it is seen that, taking a suitable cycle, a restriction of the $q$-Jordan-Pochhammer integral expresses the Macdonald polynomial of the $C_{n}$ type parametrized by the partition $(\lambda, 0, \ldots, 0)$. This integral leads to a more explicit expression.

We believe that the present paper represents a first step toward understanding the $B C_{n}$ type QKZ equation and the $B C_{n}$ type Macdonald polynomial. It is noteworthy that even in the classical $(q=1)$ case was not previously known that such an integral gives spherical functions associated with the root system $C_{n}$. For related works on $B C_{n}$ type spherical functions, we refer the reader to [6] and references therein.

Throughout this paper, $q$ is regarded as a real number satisfying $0 \leq q<1$.

\section{QKZ EQUATION OF TYPE $C_{n}$}

We first give a review of the QKZ equation associated with the root system of type $C_{n}$ for the reader's convenience, following Cherednik [2] and Kato [7].

Key words and phrases. Quantum Knizhnik Zamolodchikov equations, Macdonald polynomials, $q$-Jordan-Pochhammer integrals. 
Let $E=\oplus_{1 \leq i \leq n} \mathbb{R} \epsilon_{i}$ be the real Euclidean space with inner product $\langle$,$\rangle such$ that $\left\langle\epsilon_{i}, \epsilon_{j}\right\rangle=\delta_{i j}$. Let $\Delta=\left\{ \pm \epsilon_{i} \pm \epsilon_{j}(1 \leq i<j \leq n), \pm 2 \epsilon_{i}(1 \leq i \leq n)\right\}$ be the root system of type $C_{n}, \Delta^{+}=\left\{\epsilon_{i} \pm \epsilon_{j}(1 \leq i<j \leq n), 2 \epsilon_{i}(1 \leq i \leq n)\right\}$ the set of positive roots, $\Pi=\left\{\alpha_{i}=\epsilon_{i}-\epsilon_{i+1}(1 \leq i \leq n-1), \alpha_{n}=2 \epsilon_{n}\right\}$ the set of simple roots, $P=\oplus_{1 \leq i \leq n} \mathbb{Z} \epsilon_{i}$ the weight lattice, and $P^{\vee}=\oplus_{1 \leq i \leq n} \mathbb{Z} \epsilon_{i}+\mathbb{Z}\left(\frac{1}{2} \sum_{i=1}^{n} \epsilon_{i}\right)$ the dual weight lattice for the root system $\Delta$. We frequently write $\alpha \in \Delta^{+}$as $\alpha>0$.

An element of the group algebra $A=\mathbb{C}[P]$ is denoted by $e^{\lambda}$, as is customary. Then the Weyl group $W=W\left(C_{n}\right)=\left\langle s_{1}, s_{2}, \ldots, s_{n}\right\rangle$ (where each $s_{i}$ is a standard generator corresponding to the simple root $\left.\alpha_{i}\right)$ acts on $A$ as $w\left(e^{\lambda}\right)=e^{w \lambda}(w \in W)$. The symbol $s_{\alpha}$ denoting the reflections is defined by $s_{\alpha}(x)=x-\langle x, \alpha\rangle \alpha^{\vee}$, with $\alpha^{\vee}=2 \alpha /\langle\alpha, \alpha\rangle$ for $x \in E$ and $\alpha \in \Delta$.

The set of affine roots associated with $\Delta$ is $\tilde{\Delta}=\{\alpha+m \delta ; \alpha \in \Delta, m \in \mathbb{Z}\}$, where $\delta$ denotes the constant function 1 on $E$. The simple roots are $a_{0}=-\theta+\delta$ with the highest root $\theta=2 \epsilon_{1}$ and $a_{i}=\alpha_{i} \in \Delta$ for $1 \leq i \leq n$. We use the symbol introduced above, $s_{i}(0 \leq i \leq n)$ to also represent the generator for the corresponding affine Weyl group. We note that $s_{0}=\tau\left(\theta^{\vee}\right) s_{\theta}=\tau\left(\epsilon_{1}\right) s_{2 \epsilon_{1}}$, where $\tau(\mu)$ is a translation by $\mu$.

Let us introduce $V$ as the left free $A$-module of rank $|W|=2^{n} n$ ! with the free basis $h_{w}(w \in W)$; each element $F$ of $V$ can be written uniquely as $F=$ $\sum_{w \in W} f_{w} h_{w}\left(f_{w} \in A\right)$. Then, let $A^{\sim}$ be a completion of the quotient field of $A$. We then have $V^{\sim}=A^{\sim} \otimes_{A} V$. The action $r_{w}$ of the Weyl group $W$ on $V^{\sim}$ is defined by the following:

$$
r_{w}\left(f h_{y}\right)=w(f) h_{w y} \quad \text { for } \quad f \in A \quad \text { and } \quad w, y \in W .
$$

Moreover, the action of the translation $\tau(\mu)\left(\mu \in P^{\vee}\right)$ for a parameter $u \in E$ is given by

$$
\tau(\mu) e^{\lambda}=q^{-\langle\lambda, \mu\rangle} e^{\lambda} \quad \text { for } \quad \lambda \in P, \quad \tau(\mu) h_{w}=q^{\langle\mu, w u\rangle} h_{w} \quad \text { for } \quad w \in W
$$

and

$$
r_{\tau(\mu)}\left(f h_{w}\right)=\tau(\mu)(f) q^{\langle\mu, w u\rangle} h_{w} \quad \text { for } \quad f \in A \quad \text { and } \quad w \in W .
$$

This is an evaluation representation for which $e^{\delta}$ is identified with $q$.

Hereafter the symbol $r_{w}$ is used also to represent the element $w$ from the extended affine Weyl group $W_{P^{\vee}}=W \ltimes P^{\vee}$ (the semidirect product of $W$ and $P^{\vee}$ ). Then $r_{w} \tau\left(\epsilon_{i}\right)=\tau\left(w\left(\epsilon_{i}\right)\right) r_{w}$. Note also that, if $w=v \tau(\lambda), v \in W, \lambda \in P^{\vee}$, we have $w(\mu)=v \mu-\langle\lambda, \mu\rangle \delta$ for $\mu \in P$.

For an affine root $\alpha+m \delta(\alpha \in \Delta, m \in \mathbb{Z})$, define the $R$-matrix $R_{\alpha+m \delta}$ as an element of $\operatorname{End} d_{A}\left(V^{\sim}\right)$ by the formula

$$
R_{\alpha+m \delta} h_{y}= \begin{cases}a_{\alpha+m \delta} h_{y}+q^{m\left\langle\alpha^{\vee}, y u\right\rangle} b_{\alpha+m \delta} h_{s_{\alpha} y}, & y^{-1}(\alpha)>0 \\ c_{\alpha+m \delta} h_{y}+q^{m\left\langle\alpha^{\vee}, y u\right\rangle} d_{\alpha+m \delta} h_{s_{\alpha} y}, & y^{-1}(\alpha)<0\end{cases}
$$

for $y \in W$, where

$$
\begin{aligned}
a_{\alpha+m \delta} & =\frac{1-q^{m} e^{\alpha}}{1-t_{\alpha} q^{m} e^{\alpha}}, & b_{\alpha+m \delta} & =\frac{1-t_{\alpha}}{1-t_{\alpha} q^{m} e^{\alpha}}, \\
c_{\alpha+m \delta} & =\frac{t_{\alpha}\left(1-q^{m} e^{\alpha}\right)}{1-t_{\alpha} q^{m} e^{\alpha}}, & d_{\alpha+m \delta} & =\frac{q^{m} e^{\alpha}\left(1-t_{\alpha}\right)}{1-t_{\alpha} q^{m} e^{\alpha}}
\end{aligned}
$$


and $\alpha \mapsto t_{\alpha}$ is a $W$-invariant function taking positive values; there are two different $t_{\alpha}$, which we may write as $t_{1}=t_{ \pm \epsilon_{i} \pm \epsilon_{j}}, t_{2}=t_{ \pm 2 \epsilon_{j}}$.

It is seen that

$$
\begin{aligned}
r_{w} R_{\alpha} & =R_{w(\alpha)} r_{w} \quad \text { for } \quad \alpha \in \tilde{\Delta}, w \in W_{P^{\vee}}, \\
R_{\beta} & =R_{-\beta}^{-1} \quad \text { for } \quad \beta \in \tilde{\Delta}
\end{aligned}
$$

and

$$
\left\{\begin{array}{l}
R_{\epsilon_{i}-\epsilon_{j}} R_{\epsilon_{i}-\epsilon_{k}} R_{\epsilon_{j}-\epsilon_{k}}=R_{\epsilon_{j}-\epsilon_{k}} R_{\epsilon_{i}-\epsilon_{k}} R_{\epsilon_{i}-\epsilon_{j}}, \quad 1 \leq i<j<k \leq n, \\
R_{\epsilon_{i}-\epsilon_{j}} R_{2 \epsilon_{i}} R_{\epsilon_{i}+\epsilon_{j}} R_{2 \epsilon_{j}}=R_{2 \epsilon_{j}} R_{\epsilon_{i}+\epsilon_{j}} R_{2 \epsilon_{i}} R_{\epsilon_{i}-\epsilon_{j}}, \quad 1 \leq i<j \leq n .
\end{array}\right.
$$

The relations in (2.3) constitute the Yang-Baxter equation associated with the root system of type $C_{n}$.

Then we can state the definition of the QKZ equation for the root system of type $C_{n}$.

Definition 2.1. The $Q K Z$ equation for the root system $C_{n}$ with a parameter $u=$ $\left(u_{1}, \ldots, u_{n}\right) \in \mathbb{R}^{n}$ is the following system of equations:

$$
r_{\tau\left(\epsilon_{i}\right)}^{-1} F=R_{\tau\left(\epsilon_{i}\right)} F, \quad 1 \leq i \leq n,
$$

and

$$
r_{\tau\left(\frac{1}{2}\left(\epsilon_{1}+\cdots+\epsilon_{n}\right)\right)}^{-1} F=R_{\tau\left(\frac{1}{2}\left(\epsilon_{1}+\cdots+\epsilon_{n}\right)\right)} F
$$

for $F \in V^{\sim}$, with

$$
\begin{aligned}
R_{\tau\left(\epsilon_{i}\right)} & =R_{\epsilon_{i}-\epsilon_{i-1}+\delta} \cdots R_{\epsilon_{i}-\epsilon_{1}+\delta} R_{2 \epsilon_{i}+\delta} R_{\epsilon_{1}+\epsilon_{i}} \cdots R_{\epsilon_{i-1}+\epsilon_{i}} \\
& \times R_{\epsilon_{i}+\epsilon_{i+1}} \cdots R_{\epsilon_{i}+\epsilon_{n}} R_{2 \epsilon_{i}} R_{\epsilon_{i}-\epsilon_{n}} \cdots R_{\epsilon_{i}-\epsilon_{i+1}}
\end{aligned}
$$

for $1 \leq i \leq n$, and

$$
\begin{aligned}
R_{\tau\left(\frac{1}{2}\left(\epsilon_{1}+\cdots+\epsilon_{n}\right)\right)} & =\left(R_{2 \epsilon_{1}} R_{\epsilon_{1}+\epsilon_{2}} R_{\epsilon_{1}+\epsilon_{3}} \cdots R_{\epsilon_{1}+\epsilon_{n}}\right) \\
& \times\left(R_{2 \epsilon_{2}} R_{\epsilon_{2}+\epsilon_{3}} \cdots R_{\epsilon_{2}+\epsilon_{n}}\right) \times \cdots \times\left(R_{2 \epsilon_{n-1}} R_{\epsilon_{n-1}+\epsilon_{n}}\right) R_{2 \epsilon_{n}} .
\end{aligned}
$$

Remark. If we introduce the operators $L_{\mu}\left(\mu \in P^{\vee}\right)$ and $P_{\mu}^{u} \in \operatorname{End}_{A^{\sim}}\left(V^{\sim}\right)(\mu \in$ $P^{\vee}, u \in E$ ) defined by

$$
L_{\mu}\left(\sum f_{w} h_{w}\right)=\sum L_{\mu}\left(f_{w}\right) h_{w} \quad \text { with } \quad L_{\mu}\left(e^{\lambda}\right)=q^{\langle\mu, \lambda\rangle} e^{\lambda} \quad(\lambda \in P)
$$

and

$$
P_{\mu}^{u}\left(h_{w}\right)=q^{\langle\mu, w u\rangle} h_{w}
$$

then the equation above can be rewritten as

$$
L_{\epsilon_{i}} F=P_{\epsilon_{i}}^{u} R_{\tau\left(\epsilon_{i}\right)} F
$$

and

$$
L_{\frac{1}{2}\left(\epsilon_{1}+\cdots+\epsilon_{n}\right)} F=P_{\frac{1}{2}\left(\epsilon_{1}+\cdots+\epsilon_{n}\right)}^{u} R_{\tau\left(\frac{1}{2}\left(\epsilon_{1}+\cdots+\epsilon_{n}\right)\right)} F .
$$

Fulfilment of the compatibility condition of the QKZ equation is guaranteed by the Yang-Baxter equation (2.3). 
In the next section, we will construct a solution of the QKZ equation for the special case $u=-\lambda \epsilon_{1}(\lambda>0)$ through application of the $q$-Jordan-Pochhammer integral.

\section{INTEGRALS AND MAIN RESULT}

We introduce the form

$$
\Phi=x^{\lambda} \prod_{1 \leq j \leq n} \frac{\left(t y_{j} / x\right)_{\infty}\left(t y_{j}^{-1} / x\right)_{\infty}}{\left(y_{j} / x\right)_{\infty}\left(y_{j}^{-1} / x\right)_{\infty}} \frac{d x}{x}
$$

where $(a)_{\infty}=\prod_{s>0}\left(1-a q^{s}\right)$. This can be regarded as a form of a restriction of the $q$-Jordan-Pochhammer integral

$$
x^{\lambda} \prod_{1 \leq j \leq 2 n} \frac{\left(t y_{j} / x\right)_{\infty}}{\left(y_{j} / x\right)_{\infty}} \frac{d x}{x}
$$

which is studied in [14] and [1].

Next, to construct our solution in case of $u=-\lambda \epsilon_{1}(\lambda>0)$, we use the induced representaion of the Weyl group $W=W\left(C_{n}\right)$ from the trivial representation of a parabolic subgroup.

As a parabolic subgroup of $W$, we choose a stabilizer $W_{\epsilon_{1}}=\left\langle s_{2}, \ldots, s_{n}\right\rangle$ of $\epsilon_{1}$. A representative of the quotient $W / W_{\epsilon_{1}}$ is fixed to be

$$
\left\{\begin{array}{l}
w_{1}=e, w_{2}=s_{1}, w_{3}=s_{2} s_{1}, \ldots, w_{n+1}=s_{n} \cdots s_{2} s_{1}, \\
w_{n+2}=s_{n-1} w_{n+1}, w_{n+3}=s_{n-2} s_{n-1} w_{n+1}, \ldots, w_{2 n}=s_{1} \cdots s_{n-1} w_{n+1} .
\end{array}\right.
$$

It is seen that the element $\bar{h}_{e}=\sum_{g \in W_{\epsilon_{1}}} h_{g}$ is invariant under the action of $W_{\epsilon_{1}}$ and the induced representation of $W$ from $\bar{h}_{e}$ is given by the elements

$$
\bar{h}_{w_{i}}=\sum_{g \in W_{\epsilon_{1}}} h_{w_{i} g} \quad(1 \leq i \leq 2 n) .
$$

Using $w_{i}$ as suffices, we define the following rational functions:

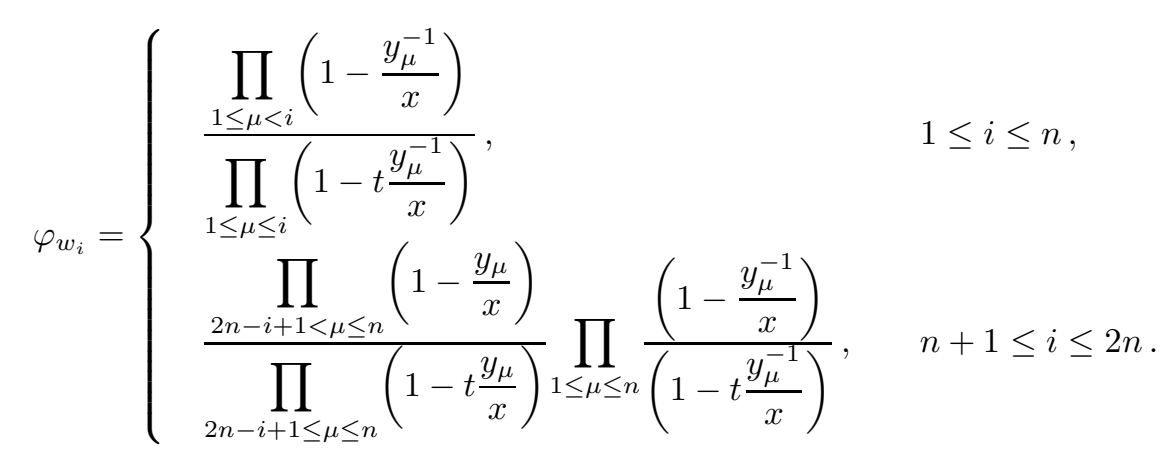

Associated with the function $\Phi$, we write

$$
\langle\psi\rangle=\int_{\mathcal{C}} \psi \Phi
$$

for a rational function $\psi$ and a fixed cycle $\mathcal{C}$, and define the element $\Psi$ by

$$
\Psi=\sum_{1 \leq i \leq 2 n}\left\langle\varphi_{w_{i}}\right\rangle \bar{h}_{w_{i}}
$$


Then we obtain the following, which will be proven in the next section.

Proposition 3.1. $r_{a_{i}} \Psi=R_{a_{i}} \Psi \quad$ for $\quad 0 \leq i \leq n$.

We are now in a position to state our main result.

Theorem 3.2. The function

$$
\Psi=\sum_{1 \leq i \leq 2 n}\left\langle\varphi_{w_{i}}\right\rangle \bar{h}_{w_{i}}
$$

satisfies the $Q K Z$ equation of type $C_{n}$ with the parameter $u=-\lambda \epsilon_{1}(\lambda>0)$ and $t_{1}=t_{2}=t$ :

$$
r_{\tau\left(\epsilon_{i}\right)}^{-1} \Psi=R_{\tau\left(\epsilon_{i}\right)} \Psi, \quad 1 \leq i \leq n
$$

and

$$
r_{\tau\left(\frac{1}{2}\left(\epsilon_{1}+\cdots+\epsilon_{n}\right)\right)}^{-1} \Psi=R_{\tau\left(\frac{1}{2}\left(\epsilon_{1}+\cdots+\epsilon_{n}\right)\right)} \Psi .
$$

From this point we use the identification $y_{i}=e^{\epsilon_{i}}$ for $1 \leq i \leq n$.

It is seen that a system of fundamental solutions is obtained by taking suitable linearly independent cycles.

Proof. We first note

$$
r_{\tau\left(\epsilon_{1}\right)}^{-1} \Psi=r_{s_{\theta} s_{0}} \Psi
$$

Proposition 3.1 and (2.1) imply

$$
r_{s_{\theta} s_{0}} \Psi=r_{s_{\theta}} r_{s_{0}} \Psi=r_{s_{\theta}} R_{\alpha_{0}} \Psi=R_{s_{\theta}\left(\alpha_{0}\right)} r_{s_{\theta}} \Psi .
$$

Applying this process repeatedly, we finally obtain

$$
\begin{aligned}
r_{s_{\theta} s_{0}} \Psi= & R_{s_{\theta}\left(\alpha_{0}\right)} R_{\left(s_{1} \cdots s_{n}\right)\left(s_{n-1} \cdots s_{2}\right)\left(\alpha_{1}\right)} R_{\left(s_{1} \cdots s_{n}\right)\left(s_{n-1} \cdots s_{3}\right)\left(\alpha_{2}\right)} \cdots R_{\left(s_{1} \cdots s_{n}\right)\left(\alpha_{n-1}\right)} \\
& \times R_{\left(s_{1} \cdots s_{n-1}\right)\left(\alpha_{n}\right)} \cdots R_{s_{1}\left(\alpha_{2}\right)} R_{\alpha_{1}} \Psi \\
= & R_{2 \epsilon_{1}+\delta} R_{\epsilon_{1}+\epsilon_{2}} \cdots R_{\epsilon_{1}+\epsilon_{n}} R_{2 \epsilon_{1}} R_{\epsilon_{1}-\epsilon_{n}} \cdots R_{\epsilon_{1}-\epsilon_{2}} \Psi \\
\text { since } s_{\theta}= & \left(s_{1} \cdots s_{n-1}\right)\left(s_{n} \cdots s_{1}\right) . \text { Thus we have } \\
& r_{\tau\left(\epsilon_{1}\right)}^{-1} \Psi=R_{2 \epsilon_{1}+\delta} R_{\epsilon_{1}+\epsilon_{2}} \cdots R_{\epsilon_{1}+\epsilon_{n}} R_{2 \epsilon_{1}} R_{\epsilon_{1}-\epsilon_{n}} \cdots R_{\epsilon_{1}-\epsilon_{2}} \Psi
\end{aligned}
$$

Next, let us apply $r_{s_{i-1} \cdots s_{1}}$ on both sides of (3.4). Then the left-hand side is

$$
\begin{aligned}
& r_{s_{i-1} \cdots s_{1}} r_{\tau\left(\epsilon_{1}\right)}^{-1} \Psi=r_{\tau\left(s_{i-1} \cdots s_{1}\left(\epsilon_{1}\right)\right)}^{-1} r_{s_{i-1} \cdots s_{1}} \Psi \\
& =r_{\tau\left(s_{i-1} \cdots s_{1}\left(\epsilon_{1}\right)\right)}^{-1} R_{s_{i-1} \cdots s_{2}\left(\alpha_{1}\right)} R_{s_{i-1} \cdots s_{3}\left(\alpha_{2}\right)} \cdots R_{s_{i-1}\left(\alpha_{i-2}\right)} R_{\alpha_{i-1}} \Psi \\
& =r_{\tau\left(\epsilon_{i}\right)}^{-1} R_{\epsilon_{1}-\epsilon_{i}} R_{\epsilon_{2}-\epsilon_{i}} \cdots R_{\epsilon_{i-2}-\epsilon_{i}} R_{\epsilon_{i-1}-\epsilon_{i}} \Psi \\
& =R_{\epsilon_{1}-\epsilon_{i}-\delta} R_{\epsilon_{2}-\epsilon_{i}-\delta} \cdots R_{\epsilon_{i-2}-\epsilon_{i}-\delta} R_{\epsilon_{i-1}-\epsilon_{i}-\delta} r_{\tau\left(\epsilon_{i}\right)}^{-1} \Psi .
\end{aligned}
$$

This follows from the relation $\tau\left(-\epsilon_{i}\right)\left(\epsilon_{j}-\epsilon_{i}\right)=\epsilon_{j}-\epsilon_{i}-\delta$.

On the other hand, the right-hand side is

$$
\begin{aligned}
& r_{s_{i-1} \cdots s_{1}} R_{2 \epsilon_{1}+\delta} R_{\epsilon_{1}+\epsilon_{2}} \cdots R_{\epsilon_{1}+\epsilon_{n}} R_{2 \epsilon_{1}} R_{\epsilon_{1}-\epsilon_{n}} \cdots R_{\epsilon_{1}-\epsilon_{2}} \Psi \\
= & R_{2 \epsilon_{i}+\delta} R_{\epsilon_{1}+\epsilon_{i}} R_{\epsilon_{2}+\epsilon_{i}} \cdots R_{\epsilon_{i-1}+\epsilon_{i}} R_{\epsilon_{i}+\epsilon_{i+1}} \cdots R_{\epsilon_{i}+\epsilon_{n}} R_{2 \epsilon_{i}} \Psi .
\end{aligned}
$$

Here we have used

$$
r_{s_{i-1} \cdots s_{1}}^{-1} \Psi=R_{\epsilon_{i}-\epsilon_{n}} \cdots R_{\epsilon_{n-1}-\epsilon_{n}} \Psi
$$


Therefore we reach the desired relation (3.2) by using (2.2).

Next we proceed to derive (3.3).

For $1 \leq i \leq n$, we have

$$
\begin{aligned}
& r_{\tau\left(\frac{1}{2}\left(\epsilon_{1}+\cdots+\epsilon_{n}\right)\right)}^{-1}\left\langle\varphi_{w_{i}}\right\rangle \\
& =\int_{C} x^{\lambda} \prod_{k=1}^{n} \frac{\left(q^{\frac{1}{2}} \frac{t y_{k}}{x}\right)_{\infty}}{\left(q^{\frac{1}{2}} \frac{y_{k}}{x}\right)_{\infty}} \frac{\prod_{k=i+1}^{n}\left(q^{-\frac{1}{2}} \frac{t y_{k}^{-1}}{x}\right)_{\infty} \prod_{k=1}^{i}\left(q^{\frac{1}{2}} \frac{t y_{k}^{-1}}{x}\right)_{\infty}}{\prod_{k=i}^{n}\left(q^{-\frac{1}{2}} \frac{y_{k}^{-1}}{x}\right)_{\infty} \prod_{k=1}^{i-1}\left(q^{\frac{1}{2}} \frac{y_{k}^{-1}}{x}\right)_{\infty}} \frac{d x}{x}
\end{aligned}
$$

and

$$
\begin{aligned}
& r_{\tau\left(\frac{1}{2}\left(\epsilon_{1}+\cdots+\epsilon_{n}\right)\right)}^{-1}\left\langle\varphi_{w_{n+i}}\right\rangle \\
& =\int_{C} x^{\lambda} \frac{\prod_{k=1}^{n-i}\left(q^{\frac{1}{2}} \frac{t y_{k}}{x}\right)_{\infty} \prod_{k=n-i+1}^{n}\left(q^{\frac{3}{2}} \frac{t y_{k}}{x}\right)_{\infty}}{\prod_{k=1}^{n-i+1}\left(q^{\frac{1}{2}} \frac{y_{k}}{x}\right)_{\infty}} \prod_{k=n-i+2}^{n}\left(q^{\frac{3}{2}} \frac{y_{k}}{x}\right)_{\infty} \frac{\left(q^{\frac{1}{2}} \frac{t y_{k}^{-1}}{x}\right)_{\infty}}{\left(q^{\frac{1}{2}} \frac{y_{k}^{-1}}{x}\right)_{\infty}} \frac{d x}{x} .
\end{aligned}
$$

By changing the integration variable such that $x \mapsto q^{-1 / 2} x$, from (3.5) we have

$$
\begin{aligned}
& r_{\tau\left(\frac{1}{2}\left(\epsilon_{1}+\cdots+\epsilon_{n}\right)\right)}^{-1}\left\langle\varphi_{w_{i}}\right\rangle \\
& =q^{-\frac{\lambda}{2}} \int_{C} x^{\lambda} \prod_{k=1}^{n} \frac{\left(q \frac{t y_{k}}{x}\right)_{\infty}}{\left(q \frac{y_{k}}{x}\right)_{\infty}} \frac{\prod_{k=i+1}^{n}\left(\frac{t y_{k}^{-1}}{x}\right)_{\infty} \prod_{k=1}^{i}\left(q \frac{t y_{k}^{-1}}{x}\right)_{\infty}\left(\frac{y_{k}^{-1}}{x}\right)_{\infty} \prod_{k=1}^{i-1}\left(q \frac{y_{k}^{-1}}{x}\right)_{\infty} \frac{d x}{x}}{=q^{-\frac{\lambda}{2}}\left\langle g \varphi_{w_{n+i}}\right\rangle}
\end{aligned}
$$

with

$$
g=s_{n}\left(s_{n-1} s_{n}\right)\left(s_{n-2} s_{n-1} s_{n}\right) \cdots\left(s_{1} \cdots s_{n}\right) \in W .
$$

Here we note $g\left(\epsilon_{i}\right)=-\epsilon_{n-i+1}$ for each $1 \leq i \leq n$.

Similarly, as a result of the change $x \mapsto q^{1 / 2} x$, from (3.6) we have

$$
\begin{aligned}
& r_{\tau\left(\frac{1}{2}\left(\epsilon_{1}+\cdots+\epsilon_{n}\right)\right)}^{-1}\left\langle\varphi_{w_{n+i}}\right\rangle \\
& =q^{\frac{\lambda}{2}} \int_{C} x^{\lambda} \frac{\prod_{k=1}^{n-i}\left(\frac{t y_{k}}{x}\right)_{\infty} \prod_{k=n-i+1}^{n}\left(q \frac{t y_{k}}{x}\right)_{\infty}}{\prod_{k=1}^{n-i+1}\left(\frac{y_{k}}{x}\right)_{\infty}} \prod_{k=n-i+2}^{n}\left(q \frac{y_{k}}{x}\right)_{\infty} \frac{\left(\frac{t y_{k}^{-1}}{x}\right)_{\infty}}{\left(\frac{y_{k}^{-1}}{x}\right)_{\infty}} \frac{d x}{x} \\
& =q^{\frac{\lambda}{2}}\left\langle g \varphi_{w_{i}}\right\rangle
\end{aligned}
$$

with the same $g \in W$. 
As for this $g=s_{n}\left(s_{n-1} s_{n}\right)\left(s_{n-2} s_{n-1} s_{n}\right) \cdots\left(s_{1} \cdots s_{n}\right) \in W$, we have

$$
\begin{aligned}
& g w_{i}=w_{n+i} s_{n}\left(s_{n-1} s_{n}\right)\left(s_{n-2} s_{n-1} s_{n}\right) \cdots\left(s_{2} \cdots s_{n-1} s_{n}\right), \\
& g w_{n+i}=w_{i} s_{n}\left(s_{n-1} s_{n}\right)\left(s_{n-2} s_{n-1} s_{n}\right) \cdots\left(s_{2} \cdots s_{n-1} s_{n}\right)
\end{aligned}
$$

for $1 \leq i \leq n$. These relations lead to

$$
\begin{gathered}
g \bar{h}_{w_{i}}=\bar{h}_{g w_{i}}=\bar{h}_{w_{n+i}}, \\
g \bar{h}_{w_{n+i}}=\bar{h}_{g w_{n+i}}=\bar{h}_{w_{i}}
\end{gathered}
$$

for $1 \leq i \leq n$.

On the other hand, noting $u=-\lambda \epsilon_{1}$, we obtain

$$
\begin{aligned}
& \tau\left(-\frac{1}{2}\left(\epsilon_{1}+\cdots+\epsilon_{n}\right)\right) \bar{h}_{w_{i}}=q^{\left\langle-\frac{1}{2}\left(\epsilon_{1}+\cdots+\epsilon_{n}\right),-\lambda \epsilon_{1}\right\rangle} \bar{h}_{w_{i}}=q^{\frac{\lambda}{2}} \bar{h}_{w_{i}}, \\
& \tau\left(-\frac{1}{2}\left(\epsilon_{1}+\cdots+\epsilon_{n}\right)\right) \bar{h}_{w_{n+i}}=q^{\left\langle-\frac{1}{2}\left(\epsilon_{1}+\cdots+\epsilon_{n}\right), \lambda \epsilon_{n-i+1}\right\rangle} \bar{h}_{w_{n+i}}=q^{-\frac{\lambda}{2}} \bar{h}_{w_{n+i}}
\end{aligned}
$$

for $1 \leq i \leq n$.

Combining these relations, we get

$$
\begin{aligned}
& \tau\left(-\frac{1}{2}\left(\epsilon_{1}+\cdots+\epsilon_{n}\right)\right) \Psi \\
= & \tau\left(-\frac{1}{2}\left(\epsilon_{1}+\cdots+\epsilon_{n}\right)\right) \sum_{1 \leq i \leq n}\left\{\left\langle\varphi_{w_{i}}\right\rangle \bar{h}_{w_{i}}+\left\langle\varphi_{w_{n+i}}\right\rangle \bar{h}_{w_{n+i}}\right\} \\
= & \sum_{1 \leq i \leq n}\left\{q^{-\frac{\lambda}{2}}\left\langle g \varphi_{w_{n+i}}\right\rangle q^{\frac{\lambda}{2}} \bar{h}_{w_{i}}+q^{\frac{\lambda}{2}}\left\langle g \varphi_{w_{i}}\right\rangle q^{-\frac{\lambda}{2}} \bar{h}_{w_{n+i}}\right\} \\
= & \sum_{1 \leq i \leq n}\left\{\left\langle g \varphi_{w_{n+i}}\right\rangle \bar{h}_{w_{i}}+\left\langle g \varphi_{w_{i}}\right\rangle \bar{h}_{w_{n+i}}\right\} \\
= & \sum_{1 \leq i \leq n}\left\{\left\langle g \varphi_{w_{n+i}}\right\rangle g \bar{h}_{w_{n+i}}+\left\langle g \varphi_{w_{i}}\right\rangle g \bar{h}_{w_{i}}\right\} \\
= & r_{g} \Psi
\end{aligned}
$$

At this stage, applying the relation

$$
\begin{aligned}
& r_{\left(s_{n}\left(s_{n-1} s_{n}\right) \cdots\left(s_{k+1} \cdots s_{n}\right)\right) s_{k} \cdots s_{n}} \Psi \\
& =R_{\left(s_{n}\left(s_{n-1} s_{n}\right) \cdots\left(s_{k+1} \cdots s_{n}\right)\right) s_{k} \cdots s_{n-1}\left(\alpha_{n}\right)} R_{\left(s_{n}\left(s_{n-1} s_{n}\right) \cdots\left(s_{k+1} \cdots s_{n}\right)\right) s_{k} \cdots s_{n-2}\left(\alpha_{n-1}\right)} \\
& \times \cdots \times R_{\left(s_{n}\left(s_{n-1} s_{n}\right) \cdots\left(s_{k+1} \cdots s_{n}\right)\right) s_{k}\left(\alpha_{k+1}\right)} R_{\left(s_{n}\left(s_{n-1} s_{n}\right) \cdots\left(s_{k+1} \cdots s_{n}\right)\right)\left(\alpha_{k}\right)} \\
& \times r_{\left(s_{n}\left(s_{n-1} s_{n}\right) \cdots\left(s_{k+1} \cdots s_{n-1} s_{n}\right)\right)} \Psi \\
& =R_{2 \epsilon_{k}} R_{\epsilon_{k}+\epsilon_{k+1}} \cdots R_{\epsilon_{k}+\epsilon_{n-1}} R_{\epsilon_{k}+\epsilon_{n}} \\
& \times r_{\left(s_{n}\left(s_{n-1} s_{n}\right) \cdots\left(s_{k+1} \cdots s_{n-1} s_{n}\right)\right)} \Psi, \quad(1 \leq k \leq n)
\end{aligned}
$$

repeatedly, we finally obtain

$$
\begin{aligned}
r_{g} \Psi & =\left(R_{2 \epsilon_{1}} R_{\epsilon_{1}+\epsilon_{2}} \cdots R_{\epsilon_{1}+\epsilon_{n}}\right)\left(R_{2 \epsilon_{2}} R_{\epsilon_{2}+\epsilon_{3}} \cdots R_{\epsilon_{2}+\epsilon_{n}}\right) \\
& \times \cdots \times\left(R_{2 \epsilon_{n-1}} R_{\epsilon_{n-1}+\epsilon_{n}}\right) R_{2 \epsilon_{n-1}} \Psi .
\end{aligned}
$$

Therefore, we reach the desired result (3.3). 


\section{Proof of Proposition 3.1}

To prove Proposition 3.1, we start by considering the action of $s_{i} \in W$ on the $\varphi_{w_{k}}$.

Lemma 4.1. (a) If $1 \leq i \leq n-1, s_{i} \varphi_{w_{k}}=\varphi_{w_{k}}$ for each $1 \leq k \leq 2 n$ such that $k \neq i, i+1,2 n-i, 2 n-i+1$.

(b) $s_{n} \varphi_{w_{k}}=\varphi_{w_{k}}$ for each $1 \leq k \leq 2 n$ such that $k \neq n, n+1$.

(c) $s_{0} \varphi_{w_{k}}=\varphi_{w_{k}}$ for each $1 \leq k \leq 2 n$ such that $k \neq 1,2 n$.

Proof. These assertions follow from the definition of $s_{i}$ and $\varphi_{w_{k}}$.

Moreover we have

Lemma 4.2. (a) For $1 \leq i \leq n-1$;

$$
\begin{cases}s_{i} \varphi_{w_{i+1}} & =a_{\alpha_{i}} \varphi_{w_{i}}+d_{\alpha_{i}} \varphi_{w_{i+1}} \\ s_{i} \varphi_{w_{i}} & =b_{\alpha_{i}} \varphi_{w_{i}}+c_{\alpha_{i}} \varphi_{w_{i+1}}\end{cases}
$$

and

$$
\begin{cases}s_{i} \varphi_{w_{2 n-i+1}} & =a_{\alpha_{i}} \varphi_{w_{2 n-i}}+d_{\alpha_{i}} \varphi_{w_{2 n-i+1}} \\ s_{i} \varphi_{w_{2 n-i}} & =b_{\alpha_{i}} \varphi_{w_{2 n-i}}+c_{\alpha_{i}} \varphi_{w_{2 n-i+1}}\end{cases}
$$

(b)

$$
\left\{\begin{array}{l}
s_{n} \varphi_{w_{n+1}}=a_{\alpha_{n}} \varphi_{w_{n}}+d_{\alpha_{n}} \varphi_{w_{n+1}}, \\
s_{n} \varphi_{w_{n}}=b_{\alpha_{n}} \varphi_{w_{n}}+c_{\alpha_{n}} \varphi_{w_{n+1}} .
\end{array}\right.
$$

Proof. By direct calculation or expansion of partial fractions, we find

$$
\begin{aligned}
& \frac{1-\frac{y_{i+1}^{-1}}{x}}{\left(1-t \frac{y_{i}^{-1}}{x}\right)\left(1-t \frac{y_{i+1}^{-1}}{x}\right)} \\
& =a_{\alpha_{i}} \frac{1}{1-t \frac{y_{i}^{-1}}{x}}+d_{\alpha_{i}} \frac{1-\frac{y_{i}^{-1}}{x}}{\left(1-t \frac{y_{i+1}^{-1}}{x}\right)\left(1-t \frac{y_{i}^{-1}}{x}\right)}
\end{aligned}
$$

and

$$
\frac{1}{1-t \frac{y_{i+1}^{-1}}{x}}=b_{\alpha_{i}} \frac{1}{1-t \frac{y_{i}^{-1}}{x}}+c_{\alpha_{i}} \frac{1-\frac{y_{i}^{-1}}{x}}{\left(1-t \frac{y_{i+1}^{-1}}{x}\right)\left(1-t \frac{y_{i}^{-1}}{x}\right)} .
$$

Multiplying the factor

$$
\prod_{j=1}^{i-1} \frac{1-\frac{y_{j}^{-1}}{x}}{1-t \frac{y_{j}^{-1}}{x}}
$$

on both sides of each equality (4.4) or (4.5), we get the desired relations (4.1). 
While the change of variables $\epsilon_{i} \mapsto-\epsilon_{i+1}$ and $\epsilon_{i+1} \mapsto-\epsilon_{i}$ leave $\alpha_{i}$ unchanged, they produce the following from (4.4) and (4.5):

$$
\begin{aligned}
& \frac{1-\frac{y_{i}}{x}}{\left(1-t \frac{y_{i}}{x}\right)\left(1-t \frac{y_{i+1}}{x}\right)} \\
& =a_{\alpha_{i}} \frac{1}{1-t \frac{y_{i+1}}{x}}+d_{\alpha_{i}} \frac{1-\frac{y_{i+1}}{x}}{\left(1-t \frac{y_{i}}{x}\right)\left(1-t \frac{y_{i+1}}{x}\right)}
\end{aligned}
$$

and

$$
\frac{1}{1-t \frac{y_{i}}{x}}=b_{\alpha_{i}} \frac{1}{1-t \frac{y_{i+1}}{x}}+c_{\alpha_{i}} \frac{1-\frac{y_{i+1}}{x}}{\left(1-t \frac{y_{i}}{x}\right)\left(1-t \frac{y_{i+1}}{x}\right)} .
$$

Multiplying the factor

$$
\prod_{j=i+2}^{n} \frac{1-\frac{y_{j}}{x}}{1-t \frac{y_{j}}{x}} \prod_{j=1}^{n} \frac{1-\frac{y_{j}^{-1}}{x}}{1-t \frac{y_{j}^{-1}}{x}}
$$

on both sides of equalities (4.6) and (4.7), we obtain the desired relations (4.2).

Similarly, changing $\epsilon_{i} \mapsto \epsilon_{n}$ and $\epsilon_{i+1} \mapsto-\epsilon_{n}$ induces $\alpha_{i} \mapsto \alpha_{n}$ and leads from equalities (4.4) and (4.5) to

$$
\begin{aligned}
& \frac{1-\frac{y_{n}}{x}}{\left(1-t \frac{y_{n}}{x}\right)\left(1-t \frac{y_{n}^{-1}}{x}\right)} \\
& =a_{\alpha_{n}} \frac{1}{1-t \frac{y_{n}^{-1}}{x}}+d_{\alpha_{n}} \frac{1-\frac{y_{n}^{-1}}{x}}{\left(1-t \frac{y_{n}}{x}\right)\left(1-t \frac{y_{n}^{-1}}{x}\right)}
\end{aligned}
$$

and

$$
\frac{1}{1-t \frac{y_{n}}{x}}=b_{\alpha_{n}} \frac{1}{1-t \frac{y_{n}^{-1}}{x}}+c_{\alpha_{n}} \frac{1-\frac{y_{n}^{-1}}{x}}{\left(1-t \frac{y_{n}}{x}\right)\left(1-t \frac{y_{n}^{-1}}{x}\right)} .
$$

Multiplying the factor

$$
\prod_{j=1}^{n-1} \frac{1-\frac{y_{j}^{-1}}{x}}{1-t \frac{y_{j}^{-1}}{x}}
$$

on both sides of equalities (4.8) and (4.9), we get the desired relations (4.3).

In contrast to the action of $s_{i}$ for $1 \leq i \leq n$, the action of $s_{0}$ is understood as it acts on the $q$ de Rham cohomology, not on the rational functions. 
Lemma 4.3.

$$
\begin{cases}q^{\lambda}\left\langle s_{0} \varphi_{w_{1}}\right\rangle & =a_{\delta-\theta}\left\langle\varphi_{w_{2 n}}\right\rangle+q^{\lambda} d_{\delta-\theta}\left\langle\varphi_{w_{1}}\right\rangle \\ q^{-\lambda}\left\langle s_{0} \varphi_{w_{2 n}}\right\rangle & =q^{-\lambda} b_{\delta-\theta}\left\langle\varphi_{w_{2 n}}\right\rangle+c_{\delta-\theta}\left\langle\varphi_{w_{1}}\right\rangle .\end{cases}
$$

Proof. Make the change of variables $\epsilon_{i} \mapsto-\epsilon_{1}$ and $\epsilon_{i+1} \mapsto \epsilon_{1}-\delta$ (i.e. $y_{i}^{-1} \mapsto$ $\left.y_{1}, y_{i+1}^{-1} \mapsto q y_{1}^{-1}\right)$ in (4.4) and (4.5). Then we have

$$
\begin{aligned}
& \frac{1-q \frac{y_{1}^{-1}}{x}}{\left(1-t \frac{y_{1}}{x}\right)\left(1-t q \frac{y_{1}^{-1}}{x}\right)} \\
& =a_{\delta-\theta} \frac{1}{1-t \frac{y_{1}}{x}}+d_{\delta-\theta} \frac{1-\frac{y_{1}}{x}}{\left(1-t q \frac{y_{1}^{-1}}{x}\right)\left(1-t \frac{y_{1}}{x}\right)}
\end{aligned}
$$

and

$$
\frac{1}{1-t q \frac{y_{1}^{-1}}{x}}=b_{\delta-\theta} \frac{1}{1-t \frac{y_{1}}{x}}+c_{\delta-\theta} \frac{1-\frac{y_{1}}{x}}{\left(1-t q \frac{y_{1}^{-1}}{x}\right)\left(1-t \frac{y_{1}}{x}\right)} .
$$

Integration after multiplying the factor

$$
\prod_{j=2}^{n} \frac{1-\frac{y_{j}}{x}}{1-t \frac{y_{j}}{x}} \prod_{j=1}^{n} \frac{1-\frac{y_{j}^{-1}}{x}}{1-t \frac{y_{j}^{-1}}{x}} \Phi
$$

on both sides of equalities (4.11) and (4.12) gives the following:

$$
\begin{aligned}
& \int_{C} x^{\lambda} \frac{\prod_{k=1}^{n}\left(q \frac{t y_{k}}{x}\right)_{\infty}}{\left(\frac{y_{1}}{x}\right)_{\infty} \prod_{k=2}^{n}\left(q \frac{y_{k}}{x}\right)_{\infty}} \frac{\left(q^{2} \frac{t y_{1}^{-1}}{x}\right)_{\infty}}{\left(q^{2} \frac{y_{1}^{-1}}{x}\right)_{\infty}} \prod_{k=2}^{n} \frac{\left(q \frac{t y_{k}^{-1}}{x}\right)_{\infty}}{\left(q \frac{y_{k}^{-1}}{x}\right)_{\infty}} \frac{d x}{x} \\
& =a_{\delta-\theta} \int_{C} x^{\lambda} \frac{\prod_{k=1}^{n}\left(q \frac{t y_{k}}{x}\right)_{\infty}}{\left(\frac{y_{1}}{x}\right)_{\infty} \prod_{k=2}^{n}\left(q \frac{y_{k}}{x}\right)_{\infty}} \prod_{k=1}^{n} \frac{\left(q \frac{t y_{k}^{-1}}{x}\right)_{\infty}}{\left(q \frac{y_{k}^{-1}}{x}\right)_{\infty}} \frac{d x}{x} \\
& +d_{\delta-\theta} \int_{C} x^{\lambda} \prod_{k=1}^{n} \frac{\left(q \frac{t y_{k}}{x}\right)_{\infty}}{\left(q \frac{y_{k}}{x}\right)_{\infty}} \frac{\left(q^{2} \frac{y_{1}^{-1}}{x}\right)_{\infty} \prod_{k=2}^{n}\left(q \frac{t y_{k}^{-1}}{x}\right)_{\infty}}{\prod_{k=1}^{n}\left(q \frac{y_{k}^{-1}}{x}\right)_{\infty}} \frac{d x}{x}
\end{aligned}
$$


and

$$
\begin{aligned}
& \int_{C} x^{\lambda} \frac{\left(\frac{t y_{1}}{x}\right)_{\infty}}{\left(\frac{y_{1}}{x}\right)_{\infty}} \prod_{k=2}^{n} \frac{\left(q \frac{t y_{k}}{x}\right)_{\infty}}{\left(q \frac{y_{k}}{x}\right)_{\infty}} \frac{\left(q^{2} \frac{y_{1}^{-1}}{x}\right)_{\infty} \prod_{k=2}^{n}\left(q \frac{t y_{k}^{-1}}{x}\right)_{\infty}}{\prod_{k=1}^{n}\left(q \frac{y_{k}^{-1}}{x}\right)_{\infty}} \frac{d x}{x} \\
& =b_{\delta-\theta} \int_{C} x^{\lambda} \frac{\prod_{k=1}^{n}\left(q \frac{t y_{k}}{x}\right)_{\infty}}{\left(\frac{y_{1}}{x}\right)_{\infty} \prod_{k=2}^{n}\left(q \frac{y_{k}}{x}\right)_{\infty}} \frac{\left(q \frac{t y_{k}^{-1}}{x}\right)_{\infty}}{\left(q \frac{y_{k}^{-1}}{x}\right)_{\infty}} \frac{d x}{x} \\
& +c_{\delta-\theta} \int_{C} x^{\lambda} \prod_{k=1}^{n} \frac{\left(q \frac{t y_{k}}{x}\right)_{\infty}}{\left(q \frac{y_{k}}{x}\right)_{\infty}} \frac{\left(q^{2} \frac{y_{1}^{-1}}{x}\right)_{\infty} \prod_{k=2}^{n}\left(q \frac{t y_{k}^{-1}}{x}\right)_{\infty}}{\prod_{k=1}^{n}\left(q \frac{y_{k}^{-1}}{x}\right)_{\infty}} \frac{d x}{x}
\end{aligned}
$$

Here, changing the integration variable such that $x \mapsto q x$, we have

$$
\begin{aligned}
& \int_{C} x^{\lambda} \frac{\prod_{k=1}^{n}\left(q \frac{t y_{k}}{x}\right)_{\infty}}{\left(\frac{y_{1}}{x}\right)_{\infty} \prod_{k=2}^{n}\left(q \frac{y_{k}}{x}\right)_{\infty}} \frac{\left(q^{2} \frac{t y_{1}^{-1}}{x}\right)_{\infty}}{\left(q^{2} \frac{y_{1}^{-1}}{x}\right)_{\infty}^{n} \frac{\left(q \frac{t y_{k}^{-1}}{x}\right)_{\infty}}{\left(q \frac{y_{k}^{-1}}{x}\right)_{\infty}} \frac{d x}{x}} \\
& =q^{\lambda} \int_{C} x^{\lambda} \frac{\prod_{k=1}^{n}\left(\frac{t y_{k}}{x}\right)_{\infty}}{\left(q^{-1} \frac{y_{1}}{x}\right)_{\infty} \prod_{k=2}^{n}\left(\frac{t y_{1}^{-1}}{x}\right)_{\infty}} \frac{\left(q \frac{y_{1}^{-1}}{x}\right)_{\infty}^{n}}{\left(\frac{t y_{k}^{-1}}{x}\right)_{\infty}} \frac{d x}{\left(\frac{y_{k}^{-1}}{x}\right)_{\infty}} \frac{d x}{=q^{\lambda}\left\langle s_{0} \varphi_{w_{1}}\right\rangle}
\end{aligned}
$$

and

$$
\begin{aligned}
& \int_{C} x^{\lambda} \prod_{k=1}^{n} \frac{\left(q \frac{t y_{k}}{x}\right)_{\infty}}{\left(q \frac{y_{k}}{x}\right)_{\infty}} \frac{\left(q^{2} \frac{y_{1}^{-1}}{x}\right)_{\infty} \prod_{k=2}^{n}\left(q \frac{t y_{k}^{-1}}{x}\right)_{\infty}}{\prod_{k=1}^{n}\left(q \frac{y_{k}^{-1}}{x}\right)_{\infty}} \frac{d x}{x} \\
& =q^{\lambda} \int_{C} x^{\lambda} \prod_{k=1}^{n} \frac{\left(\frac{t y_{k}}{x}\right)_{\infty}}{\left(\frac{y_{k}}{x}\right)_{\infty}} \frac{\left(q \frac{y_{1}^{-1}}{x}\right)_{\infty} \prod_{k=2}^{n}\left(\frac{t y_{k}^{-1}}{x}\right)_{\infty}}{\prod_{k=1}^{n}\left(\frac{y_{k}^{-1}}{x}\right)_{\infty}} \frac{d x}{x} \\
& =q^{\lambda}\left\langle\varphi_{w_{1}}\right\rangle .
\end{aligned}
$$

Therefore, it is seen that (4.13) and (4.14) are equivalent to the desired relations (4.10).

Next, we consider the asction of $W$ on the $\bar{h}_{w_{k}}$. 
Lemma 4.4. (a) If $1 \leq i \leq n-1, \bar{h}_{s_{i} w_{k}}=\bar{h}_{w_{k}}$ for $k \neq i, i+1,2 n-i, 2 n-i+1$.

(b) $\bar{h}_{s_{n} w_{k}}=\bar{h}_{w_{k}}$ for $k \neq n, n+1$.

(c) $\bar{h}_{s_{\theta} w_{k}}=\bar{h}_{w_{k}}$ for $k \neq 1,2 n$.

Proof. In the case that $1 \leq i \leq n-1$, we have $s_{i} w_{k}=w_{k} s_{i}$ for $1 \leq k \leq i-1$ or $2 n-i+2 \leq k \leq 2 n$, and $s_{i} w_{k}=w_{k} s_{i+1}$ for $i+2 \leq k \leq 2 n-i-1$. These lead to the desired equalities in (a).

In the same way, the relations $s_{n} w_{k}=w_{k} s_{n} \quad(k \neq n, n+1)$ and $s_{\theta} w_{k}=$ $w_{k}\left(s_{2} \cdots s_{n-1}\right)\left(s_{n} \cdots s_{2}\right) \quad(k \neq 1,2 n)$ lead to the relations in (b) and (c).

Next we consider the action of $R_{\alpha_{i}}$ on the $\bar{h}_{w_{k}}$ :

Lemma 4.5. (a) If $1 \leq i \leq n-1, R_{\alpha_{i}} \bar{h}_{w_{k}}=\bar{h}_{w_{k}}$ for each $1 \leq k \leq 2 n$ such that $k \neq i, i+1,2 n-i, 2 n-i+1$.

(b) $R_{\alpha_{n}} \bar{h}_{w_{k}}=\bar{h}_{w_{k}}$ for each $1 \leq k \leq 2 n$ such that $k \neq n, n+1$.

(c) $R_{\delta-\theta} \bar{h}_{w_{k}}=\bar{h}_{w_{k}}$ for each $2 \leq k \leq 2 n-1$.

Proof. Since $w_{k}^{-1} \alpha_{i}=\alpha_{i}>0$ for $1 \leq k \leq i-1$ (then $i \geq 2$ ), we have

$$
\begin{aligned}
R_{\alpha_{i}} h_{w_{k}} & =a_{\alpha_{i}} h_{w_{k}}+b_{\alpha_{i}} h_{s_{i} w_{k}}, \\
R_{\alpha_{i}} h_{s_{i} w_{k}} & =c_{\alpha_{i}} h_{s_{i} w_{k}}+d_{\alpha_{i}} h_{w_{k}} .
\end{aligned}
$$

These imply

$$
R_{\alpha_{i}}\left(h_{w_{k}}+h_{s_{i} w_{k}}\right)=h_{w_{k}}+h_{s_{i} w_{k}}
$$

following from the relations $a_{\alpha_{i}}+d_{\alpha_{i}}=b_{\alpha_{i}}+c_{\alpha_{i}}=1$. Hence, noting $s_{i} w_{k}=w_{k} s_{i}$, we obtain $R_{\alpha_{i}} \bar{h}_{w_{k}}=\bar{h}_{w_{k}}$. Other cases are similarly derived.

Lemma 4.6. (a) For $1 \leq i \leq n-1$,

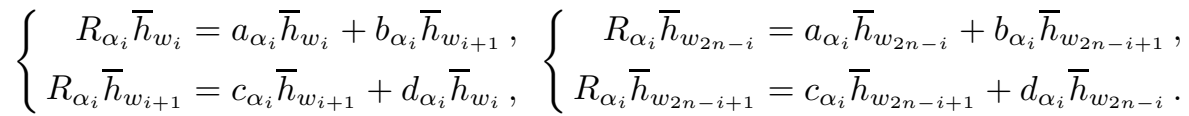

(b)

(c)

$$
\left\{\begin{aligned}
R_{\alpha_{n}} \bar{h}_{w_{n}} & =a_{\alpha_{n}} \bar{h}_{w_{n}}+b_{\alpha_{n}} \bar{h}_{w_{n+1}}, \\
R_{\alpha_{n}} \bar{h}_{w_{n+1}} & =c_{\alpha_{n}} \bar{h}_{w_{n+1}}+d_{\alpha_{n}} \bar{h}_{w_{n}} .
\end{aligned}\right.
$$

$$
\left\{\begin{aligned}
R_{\delta-\theta} \bar{h}_{w_{2 n}} & =a_{\delta-\theta} \bar{h}_{w_{2 n}}+q^{-\lambda} b_{\delta-\theta} \bar{h}_{w_{1}}, \\
R_{\delta-\theta} \bar{h}_{w_{1}} & =c_{\delta-\theta} \bar{h}_{w_{1}}+q^{\lambda} d_{\delta-\theta} \bar{h}_{w_{2 n}}
\end{aligned}\right.
$$

Proof. This follows almost immediately from the definitions.

At this stage, by combination of the above lemmas, we obtain the following:

In case of $1 \leq i \leq n-1$, we have

$$
\begin{aligned}
& r_{s_{i}} \Psi=\sum_{1 \leq k \leq 2 n}\left\langle s_{i} \varphi_{w_{k}}\right\rangle \bar{h}_{s_{i} w_{k}}=\left\{\sum_{\substack{k \neq i, i+1, 2 n-i, 2 n-i+1}}+\sum_{\substack{k=i, i+1, 2 n-i, 2 n-i+1}}\right\}\left\langle s_{i} \varphi_{w_{k}}\right\rangle \bar{h}_{s_{i} w_{k}} \\
& =\sum_{\substack{k \neq i, i+1, 2 n-i, 2 n-i+1}}\left\langle\varphi_{w_{k}}\right\rangle \bar{h}_{w_{k}} \\
& +\left\{b_{\alpha_{i}}\left\langle\varphi_{w_{i}}\right\rangle+c_{\alpha_{i}}\left\langle\varphi_{w_{i+1}}\right\rangle\right\} \bar{h}_{s_{i} w_{i}}+\left\{a_{\alpha_{i}}\left\langle\varphi_{w_{i}}\right\rangle+d_{\alpha_{i}}\left\langle\varphi_{w_{i+1}}\right\rangle\right\} \bar{h}_{s_{i} w_{i+1}}
\end{aligned}
$$




$$
\begin{aligned}
& +\left\{b_{\alpha_{i}}\left\langle\varphi_{w_{2 n-i}}\right\rangle+c_{\alpha_{i}}\left\langle\varphi_{w_{2 n-i+1}}\right\rangle\right\} \bar{h}_{s_{i} w_{2 n-i}}+\left\{a_{\alpha_{i}}\left\langle\varphi_{w_{2 n-i}}\right\rangle+d_{\alpha_{i}}\left\langle\varphi_{w_{2 n-i+1}}\right\rangle\right\} \bar{h}_{s_{i} w_{2 n-i+1}} \\
& =\sum_{\substack{k \neq i, i+1 \\
2 n-i, 2 n-i+1}}\left\langle\varphi_{w_{k}}\right\rangle \bar{h}_{w_{k}} \\
& +\left\langle\varphi_{w_{i}}\right\rangle\left\{b_{\alpha_{i}} \bar{h}_{w_{i+1}}+a_{\alpha_{i}} \bar{h}_{w_{i}}\right\}+\left\langle\varphi_{w_{i+1}}\right\rangle\left\{c_{\alpha_{i}} \bar{h}_{w_{i+1}}+d_{\alpha_{i}} \bar{h}_{w_{i}}\right\} \\
& +\left\langle\varphi_{w_{2 n-i}}\right\rangle\left\{b_{\alpha_{i}} \bar{h}_{w_{2 n-i+1}}+a_{\alpha_{i}} \bar{h}_{w_{2 n-i}}\right\}+\left\langle\varphi_{w_{2 n-i+1}}\right\rangle\left\{c_{\alpha_{i}} \bar{h}_{w_{2 n-i+1}}+d_{\alpha_{i}} \bar{h}_{w_{2 n-i+1}}\right\} \\
& =R_{\alpha_{i}} \Psi .
\end{aligned}
$$

Similarly, in the case $i=n$, we have

$$
\begin{aligned}
& r_{s_{n}} \Psi=\left\{\sum_{\substack{1 \leq k \leq 2 n \\
k \neq n, n+1}}+\sum_{k=n, n+1}\right\}\left\langle s_{n} \varphi_{w_{k}}\right\rangle \bar{h}_{s_{n} w_{k}} \\
& =\sum_{k \neq n, n+1}\left\langle\varphi_{w_{k}}\right\rangle \bar{h}_{w_{k}} \\
& +\left\{b_{\alpha_{n}}\left\langle\varphi_{w_{n}}\right\rangle+c_{\alpha_{n}}\left\langle\varphi_{w_{n+1}}\right\rangle\right\} \bar{h}_{s_{n} w_{n}}+\left\{a_{\alpha_{n}}\left\langle\varphi_{w_{n}}\right\rangle+d_{\alpha_{n}}\left\langle\varphi_{w_{n+1}}\right\rangle\right\} \bar{h}_{s_{n} w_{n+1}} \\
& =\sum_{k \neq n, n+1}\left\langle\varphi_{w_{k}}\right\rangle \bar{h}_{w_{k}} \\
& +\left\langle\varphi_{w_{n}}\right\rangle\left\{b_{\alpha_{n}} \bar{h}_{w_{n+1}}+a_{\alpha_{n}} \bar{h}_{w_{n}}\right\}+\left\langle\varphi_{w_{n+1}}\right\rangle\left\{c_{\alpha_{n}} \bar{h}_{w_{n+1}}+d_{\alpha_{n}} \bar{h}_{w_{n}}\right\} \\
& =R_{\alpha_{n}} \Psi .
\end{aligned}
$$

Finally, if $i=0$, by noting that

$$
s_{0} \bar{h}_{w_{1}}=q^{\lambda} \bar{h}_{s_{\theta} w_{1}} \quad \text { and } \quad s_{0} \bar{h}_{w_{2 n}}=q^{-\lambda} \bar{h}_{s_{\theta} w_{2 n}},
$$

we have

$$
\begin{aligned}
r_{s_{0}} \Psi= & \sum_{\substack{1 \leq k \leq 2 n \\
k \neq 1,2 n}}\left\langle\varphi_{w_{k}}\right\rangle \bar{h}_{s_{\theta} w_{k}}+\left\langle s_{0} \varphi_{w_{1}}\right\rangle q^{\lambda} \bar{h}_{s_{\theta} w_{1}}+\left\langle s_{0} \varphi_{w_{2 n}}\right\rangle q^{-\lambda} \bar{h}_{s_{\theta} w_{2 n}} \\
= & \sum_{k \neq 1,2 n}\left\langle\varphi_{w_{k}}\right\rangle \bar{h}_{w_{k}}+\left\langle s_{0} \varphi_{w_{1}}\right\rangle q^{\lambda} \bar{h}_{w_{2 n}}+\left\langle s_{0} \varphi_{w_{2 n}}\right\rangle q^{-\lambda} \bar{h}_{w_{1}} \\
= & \sum_{k \neq 1,2 n}\left\langle\varphi_{w_{k}}\right\rangle \bar{h}_{w_{k}}+\left\{c_{\delta-\theta}\left\langle\varphi_{w_{1}}\right\rangle+q^{-\lambda} b_{\delta-\theta}\left\langle\varphi_{w_{2 n}}\right\rangle\right\} \bar{h}_{w_{1}} \\
& +\left\{q^{\lambda} d_{\delta-\theta}\left\langle\varphi_{w_{1}}\right\rangle+a_{\delta-\theta}\left\langle\varphi_{w_{2 n}}\right\rangle\right\} \bar{h}_{w_{2 n}} \\
= & \sum_{k \neq 1,2 n}\left\langle\varphi_{w_{k}}\right\rangle \bar{h}_{w_{k}}+\left\langle\varphi_{w_{1}}\right\rangle\left\{c_{\delta-\theta} \bar{h}_{w_{1}}+q^{\lambda} d_{\delta-\theta} \bar{h}_{w_{2 n}}\right\} \\
& +\left\langle\varphi_{w_{2 n}}\right\rangle\left\{a_{\delta-\theta} \bar{h}_{w_{2 n}}+q^{-\lambda} b_{\delta-\theta} \bar{h}_{w_{1}}\right\} \\
= & R_{\delta-\theta} \Psi .
\end{aligned}
$$

This completes the proof of Proposition 3.1. 


\section{Macdonald POLYNomials}

Macdonald introduced the $q$-difference operators [10] to define his orthogonal polynomials associted with root sytems. In the case of a root system of type $C_{n}$, the $q$-difference operator to define such a polynomial is given by

$$
E=\sum_{a_{1}, \ldots, a_{n}= \pm 1} \prod_{1 \leq i<j \leq n} \frac{1-t y_{i}^{a_{i}} y_{j}^{a_{j}}}{1-y_{i}^{a_{i}} y_{j}^{a_{j}}} \prod_{1 \leq i \leq n} \frac{1-t y_{i}^{2 a_{i}}}{1-y_{i}^{2 a_{i}}} T_{y_{i}}^{\frac{1}{2} a_{i}}
$$

where

$$
\left(T_{y_{i}} f\right)\left(y_{1}, \ldots, y_{n}\right)=f\left(y_{1}, \ldots, q y_{i}, \ldots, y_{n}\right) .
$$

Its eigenvalue is known to be

$$
\begin{aligned}
c_{\mu} & =\sum_{a_{1}, \ldots, a_{n}= \pm 1} \prod_{j=1}^{n} q^{\frac{1}{2} \lambda_{j} a_{j}} t^{\frac{1}{2}(n-j+1) a_{j}} \\
& =q^{-\frac{1}{2}\left(\lambda_{1}+\cdots+\lambda_{n}\right)} \prod_{j=1}^{n}\left(1+t^{j} q^{\lambda_{n-j+1}}\right)
\end{aligned}
$$

with the parameter $\mu=\left(\lambda_{1}, \ldots, \lambda_{n}\right)$ (We consider only the special case corresponding to the condition $t_{1}=t_{2}=t$ ).

As for the eigenfunction of the operator $E$, we easily find the following:

Corollary 5.1. The sum

$$
\sum_{i=1}^{2 n} t^{i-1}\left\langle\varphi_{w_{i}}\right\rangle
$$

is a solution of the equation attached to the parameter $(\lambda, 0, \ldots, 0)$ :

$$
E \psi=c_{(\lambda, 0, \ldots, 0)} \psi .
$$

Proof. This is proven by applying the result of Kato (Theorem 4.6 in [7]) to our Theorem 3.2.

We next proceed to simplify the sum (5.1).

We note the equality

$$
t^{2 n} \prod_{j=1}^{n} \frac{\left(1-\frac{y_{j}}{x}\right)\left(1-\frac{y_{j}}{x}\right)}{\left(1-t \frac{y_{j}}{x}\right)\left(1-t \frac{y_{j}^{-1}}{x}\right)}=1+(t-1)\left\{\sum_{j=1}^{2 n} t^{i-1} \varphi_{w_{i}}\right\}
$$

which is demonstrated by using the partial fractions.

On the other hand, we have

$$
\left\langle\prod_{j=1}^{n} \frac{\left(1-\frac{y_{j}}{x}\right)\left(1-\frac{y_{j}}{x}\right)}{\left(1-t \frac{y_{j}}{x}\right)\left(1-t \frac{y_{j}^{-1}}{x}\right)}\right\rangle=q^{\lambda} \int_{\mathcal{C}} \Phi=q^{\lambda}\langle 1\rangle,
$$


which is demonstrated by changing the integration variable such that $x \mapsto q x$. Hence, combination of (5.3) and (5.4) gives the relation

$$
\sum_{j=1}^{2 n} t^{i-1}\left\langle\varphi_{w_{i}}\right\rangle=\frac{1-q^{\lambda} t^{2 n}}{1-t} \int_{\mathcal{C}} \Phi
$$

Therefore we reach

Proposition 5.2. The function $\int_{\mathcal{C}} \Phi$ is a solution to the equation (5.2).

It should be remarked that this is valid for arbitrary cycle $\mathcal{C}$ and that linearly independent solutions are obtained by choosing several cycles. This situation is similar to that studied in [15].

In case that the parameter $\mu$ is from the set of partitions, the eigenfunction of the form

$$
P_{\mu}(y \mid q, t)=m_{\mu}+\sum_{\nu<\mu} a_{\mu \nu} m_{\nu}
$$

is the Macdonald polynomial for the root system $C_{n}$. Here $m_{\mu}=\sum_{\nu \in W \mu} e^{\nu}$, and $\nu<\mu$ is defined to be $\mu-\nu \in Q^{+}$with $Q^{+}$the positive cone of the root lattice.

In our case, to get the Macdonald polynomial, it is enough to consider the case that $\lambda$ is a positive integer and take the cycle, with the counterclockwise direction, which encircles the sequence of poles such that $y_{i}, y_{i} q, y_{i} q^{2}, \ldots$, for $1 \leq i \leq n$ and $y_{i}^{-1}, y_{i}^{-1} q, y_{i}^{-1} q^{2}, \ldots$, for $1 \leq i \leq n$. This is an integral representaion of the Macdonald polynomial $P_{(\lambda, 0, \ldots, 0)}(y \mid q, t)$.

Moreover, applying the $q$-binomial theorem

$$
\sum_{m \geq 0} \frac{(a)_{m}}{(q)_{i}} z^{m}=\frac{(a z)_{\infty}}{(z)_{\infty}} \quad(|z|<1), \quad(a)_{m}=\prod_{0 \leq k \leq m-1}\left(1-a q^{k}\right)
$$

and the residue calculus to our integral, we obtain an exact expression of the Macdonald polynomial for the root system $C_{n}$.

\section{Theorem 5.3.}

$$
P_{(\lambda, 0, \ldots, 0)}(y \mid q, t)=\frac{(q)_{\lambda}}{(t)_{\lambda}} \sum_{\substack{i_{1}+\cdots+i_{2 n}=\lambda \\ i_{1}, \ldots, i_{2 n} \geq 0}} \frac{(t)_{i_{1}} \cdots(t)_{i_{2 n}}}{(q)_{i_{1}} \cdots(q)_{i_{2 n}}} y_{1}^{i_{1}-i_{2 n}} y_{2}^{i_{2}-i_{2 n-1}} \cdots y_{n}^{i_{n}-i_{n+1}} .
$$

Remark. We also have a dirct way to obtain the integral representation of the eigenfunction for (5.2). This will appear in a future paper. For the related work, we also refer the reader to 16 .

\section{Final comment}

We finally make a comment on the meaning of our elements $\varphi_{w_{i}}$ from the viewpoint of the Hecke algebra.

Set

$$
T_{i}=t+\frac{1-t e^{\alpha_{i}}}{1-e^{\alpha_{i}}}\left(s_{i}-1\right), \quad \text { for } \quad 1 \leq i \leq n,
$$


where $\alpha_{i}$ is an element of the simple roots and $s_{i}$ a corresponding generator of the Weyl group $W$. This is the Lusztig operator associated with the root system $C_{n}$ (in the special case $t_{1}=t_{2}=t$ ), which satisfies the following:

$$
\begin{aligned}
& \left(T_{i}-t\right)\left(T_{i}+1\right)=0 \quad(1 \leq i \leq n), \\
& T_{i} T_{i+1} T_{i}=T_{i+1} T_{i} T_{i+1} \quad(1 \leq i \leq n-2), \\
& T_{n-1} T_{n} T_{n-1} T_{n}=T_{n} T_{n-1} T_{n} T_{n-1}, \\
& T_{i} T_{j}=T_{j} T_{i} \quad(|i-j|>2) .
\end{aligned}
$$

These are the fundamental relations for the Hecke algebra $H(W)$ associated with the root system of type $C_{n}$. The action of the Lusztig operator on our $\varphi_{w_{i}}$ is given as follows.

Proposition 6.1. For $1 \leq k \leq n$;

$$
\begin{aligned}
& \left\{\begin{array}{l}
T_{i} \varphi_{w_{k}}=t \varphi_{w_{k}}, \quad i \neq k-1, k \\
T_{k-1} \varphi_{w_{k}}=(t-1) \varphi_{w_{k}}+\varphi_{w_{k-1}} \\
T_{k} \varphi_{w_{k}}=t \varphi_{w_{k+1}},
\end{array}\right. \\
& \left\{\begin{array}{l}
T_{i} \varphi_{w_{n+k}}=t \varphi_{w_{n+k}}, \quad i \neq n-k, n-k+1, \\
T_{n-k+1} \varphi_{w_{n+k}}=(t-1) \varphi_{w_{n+k}}+\varphi_{w_{n+k-1}} \\
T_{n-k} \varphi_{w_{n+k}}=t \varphi_{w_{n+k+1}} .
\end{array}\right.
\end{aligned}
$$

This shows that the vector space $\oplus_{i=1}^{2 n} \mathbb{C} \varphi_{w_{i}}$ gives the representaion of the Hecke algebra $H(W)$ for the $C_{n}$ type. Moreover, we can also obtain the representation of the affine Hecke algebra in the space of the $q$ de Rham cohomology. See 16 for $A_{n-1}$ case.

In any case, we expect that such a basis attached to the action of the Hecke algebras could be generalized to the case of higher representaions. This is our future problem.

Acknowledgement. The author wishes to thank Professor Shin-ichi Kato for valuable suggestion.

\section{REFERENCES}

[1] Aomoto, K., Kato, Y., Mimachi, K.: A solution of the Yang-Baxter equation as connection coefficients of a holonomic $q$-difference system. Internat. Math. Res. Notices 1992, No.1, $7-15$

[2] Cherednik, I.: Quantum Knizhnik-Zamolodchikov equations and affine root systems. Commun. Math. Phys. 150, 109-136 (1992)

[3] Cherednik, I.: Double affine Hecke algebras, Knizhnik-Zamolodchikov equations, and Macdonald's operators. Internat. Math. Res. Notices 1992, No.9, 171-180

[4] Cherednik, I.: Double affine Hecke algebras, and Macdonald's conjectures. Ann. of Math. 141, 191-216 (1995)

[5] Cherednik, I.: Induced representations of double affine Hecke algebras and applications. Math. Res. Lett. 1, 319-337 (1994)

[6] Debiard, A. and Gaveau, B.: Integral formulas for the spherical polynomials of a root system of type $B C_{2}$. Jour. of Funct. Anal. 119, 401-454 (1994)

[7] Kato, S.: R-matrix arising from affine Hecke algebras and its application to Macdonald's difference operators. Commun. Math. Phys. 165, 533-553 (1994)

[8] Koornwinder, T. H.: Askey-Wilson polynomials for root systems of type $B C$, in $\mathrm{Hy}$ pergeometric functions on domains of positivity, Jack polynomials and applications, D.St.P.Richards(ed.), Contemp. Math. 138, Amer. Math. Soc. (1992), pp.189-204 
[9] Lusztig, G.: Affine Hecke algebras and their graded version. J. Am. Math. Soc. 2, 599-635 (1989)

[10] Macdonald, I.G.: A new class of symmetric functions, in Actes Séminaire Lotharingen, Publ. Inst. Rech. Math. Adv., Strasbourg, 1988, 131-171

[11] Macdonald, I.G.: Affine Hecke algebras and orthogonal polynomials. Séminaire BOURBAKI, 47ème année, 1994-95, $n^{\circ} 797$

[12] Macdonald, I.G.: Symmetric Functions and Hall Polynomials (Second Edition), Oxford Mathematical Monographs, Clarendon Press, Oxford, 1995.

[13] Mimachi, K.: Connection problem in holonomic $q$-difference system associated with a Jackson integral of Jordan-Pochhammer type. Nagoya Math. J. 116, 149-161 (1989)

[14] Mimachi, K.: A solution to quantum Knizhnik-Zamolodchikov equations and its application to eigenvalue problems of the Macdonald type, Duke Math. J. 85, 635-658 (1996).

[15] Mimachi, K.: Rational solutions to eigenvalue problems of the Macdonald type, in preparation.

[16] Mimachi, K. and Noumi, M.: An integral representation of eigenfunctions for Macdonald's q-difference operators, Tôhoku Math. J. 49, 517-525 (1997).

[17] Mimachi, K. and Noumi, M.: Representaions of the Hecke algebra on a family of rational functions, preprint 1997.

Katsuhisa Mimachi

Department of Mathematics

Kyushu University 33

HAKOZAKI, FUKUOKA 812-81

JAPAN

E-mail address:mimachi@ math.kyushu-u.ac.jp 